\title{
OXIDAÇÃO INTERGRANULAR DE LIGAS DE NÍQUEL EM ALTAS TEMPERATURAS USANDO EBSD
}

Vicente Braz Trindade 1 Ulrich Krupp ${ }^{2}$

\section{Resumo}

Em geral, ligas a base de níquel exibem uma tendência ao ataque de oxidação preferencialmente ao longo de contornos de grãos da liga durante exposição a atmosferas corrosivas em altas temperaturas. Isto é devido ao maior transporte dos elementos e fácil nucleação em contornos de grão. Ligas de Ni contém elementos com alta afinidade por oxigênio, como o $\mathrm{Al}$ e o $\mathrm{Ti}$, os quais são geralmente os responsáveis pela oxidação intergranular. Estudos experimentais de oxidação das ligas de níquel Inconel ${ }^{\circledR} 718$ (UNS N077|8) e Inconel ${ }^{\circledR} 625$ (UNS N06625) foram realizados à temperatura de $850^{\circ} \mathrm{C}$ usando termogravimetria e microscopia eletrônica de varredura (MEV) em combinação com a técnica de EBSD (difração de elétrons retroespalhados). $\mathrm{O}$ efeito da difusão do oxigênio e $\mathrm{Cr}$ ao longo de contornos de grãos das ligas foi analisado através da técnica de EBSD. Os resultados suportam a hipótese de que contornos de grãos de elevada fração de sítios de rede coincidentes - CSL (baixos valores de $\Sigma$ ) parecem exibir uma maior resistência ao ataque intergranular quando comparado com contornos de grãos de altos ângulos.

Palavra-chave: Oxidação intergranular; Ligas à base de Ni; Técnica EBSD; Contornos de grãos de baixo ângulo; Difusão em contornos de grão.

\section{HIGH-TEMPERATURE INTERGRANULAR OXIDATION OF NI-BASED ALLOYS USING EBSD}

\begin{abstract}
In general, nickel-base alloys exhibit a tendency to preferred oxidation attack along the alloy grain boundaries during exposure to corrosive atmospheres at high temperatures. This is due to the much faster intergranular transport of the reacting elements in combination with easy nucleation of precipitates. Ni-base superalloys contain elements with a very high oxygen affinity, e.g., $\mathrm{Al}$ and $\mathrm{Ti}$, which are often responsible for pronounced intergranular oxidation. Experimental studies on the oxidation behaviour of Ni-base superalloys Inconel ${ }^{\circledR} 718$ (UNS N077 I8) and Inconel ${ }^{\circledR} 625$ (UNS N06625) were carried out at temperature of $850^{\circ} \mathrm{C}$ using thermogravimetry and analytical scanning electron microscopy in combination with EBSD (electron back-scattered diffraction). The effect of oxygen and $\mathrm{Cr}$ grain boundary diffusion on the overall oxidation process was analyzed by means EBSD technique. The results supported the assumption that special grain boundaries with a high fraction of coincident lattice sites (low $\Sigma$ values) seems to exhibit a higher resistance to intergranular attack as compared to random high-angle grain boundaries.
\end{abstract}

Keywords: Intergranular oxidation; Ni-based alloys; EBSD technique; Special grain boundaries; Grain boundary diffusion.

\section{INTRODUÇÃO}

Adição de cromo e alumínio é largamente usada como elementos capazes de proteger o metal base das ligas contra ataques corrosivos em altas temperaturas, através da formação de uma camada externa protetora composta de $\mathrm{Cr}_{2} \mathrm{O}_{3}$ ou $\mathrm{Al}_{2} \mathrm{O}_{3}[1,2]$. Uma subcamada de $\mathrm{SiO}_{2}$, mesmo que parcial sob camadas de $\mathrm{Cr}_{2} \mathrm{O}_{3}$ tem mostrado muito eficiente na proteção contra corrosão em altas temperaturas [2]. No entanto, mesmo com a presença de tais camadas óxidas, corrosão interna (oxidação e/ou nitretação) comumente ocorre em ligas de níquel, tendo em vista que os elementos não metálicos como oxigênio e nitrogênio conseguem difundir através da camada ou permear através de defeitos (trincas/poros) da camada e atingem o substrato com potencial químico suficiente para

'Universidade Federal de Ouro Preto - UFOP, Ouro Preto, MG, Brasil. E-mail: vicentebraz@yahoo.com.br

${ }^{2}$ University of Osnabrueck, Osnabrück, Germany.

2176-1523 (C) 2017 Associação Brasileira de Metalurgia, Materiais e Mineração. Publicado pela ABM. Este é um artigo de acesso aberto distribuído sob os termos da licença Creative Commons CC BY-NC-ND (Attribution-NonCommercial-NoDerivs) - https:// creativecommons.org/licenses/by-nc-nd/4.0\%. 
reagirem com menos nobres, como alumínio e titânio [3]. É conhecido que difusão ao longo de contornos de grãos e no grão apresentam comportamentos diferentes. Sendo a difusão ao longo de contornos de grão da ordem de $10^{6}$ vezes superior à difusão no grão. Adicionalmente, é conhecido que a difusividade nos contornos de grão depende das características do contorno de grão. Os contornos de grãos são classificados em duas categorias [4-7]: (i) contornos de alto ângulo (random grain boudaries), altos valores de $\Sigma$ e (ii) contornos de baixo ângulos (special grain boundaries), que são caracterizados por baixos valores de $\Sigma$. Sendo $\Sigma$ o valor inverso da densidade de sítios coincidentes da rede (coincident site lattice - CSL). Dados experimentais confirmam que contornos de grão com $\Sigma<29$ apresentam propriedades especiais, como por exemplo, baixa difusividade [8-10]. Portanto, as características do contorno de grão devem exercer um importante papel na corrosão intergranular, como a oxidação.

Neste artigo, duas ligas de níquel foram oxidadas em altas temperaturas e analisadas usando microscopia eletrônica de varredura (MEV) com EBSD acoplado, objetivando entender a relação entre as características dos contornos de grãos com a resistência à oxidação intergranular.

\section{MATERIAIS E MÉTODOS}

Duas ligas de $\mathrm{Ni}$ foram utilizadas neste estudo: Inconel ${ }^{\circledast} 625$ e Inconel ${ }^{\circledR} 718^{*}$. As composições químicas, em \% em massa, estão mostradas na Tabela I. Vale salientar para a composição de três importantes elementos. $\mathrm{O} \mathrm{Cr}$ que é responsável para a formação da camada óxida externa, o Al que é o elemento menos nobre, responsável pela formação da oxidação interna, em especial oxidação intergranular, e o Si que tem papel importante na proteção contra oxidação do metal de base.

Os experimentos de oxidação foram realizado a $850^{\circ} \mathrm{C}$ durante $100 \mathrm{~h}$ em uma atmosfera contendo a mistura gasosa $\mathrm{He}+\mathrm{IOvol} . \% \mathrm{O}_{2}+\mathrm{Ivol}_{2} \mathrm{HH}_{2} \mathrm{O}$. Um forno de $\mathrm{SiC}$ aquecido por resistência elétrica foi utilizado. A Figura I mostra um desenho esquemático do aparato experimental utilizado. Após geração do vapor de água, a mistura gasosa foi aquecida a uma temperatura acima do ponto de orvalho desejado. Um termo-higrômetro foi utilizado para medir o ponto de orvalho logo na entrada da mistura gasosa na parte inferior do forno.

Corpos de prova com dimensões de $10 \times 10 \times 3 \mathrm{~mm}^{3}$ foram cortados e um orifício de $1 \mathrm{~mm}$ de diâmetro usado para suspender o corpo de prova na termobalança utilizando um fino fio de quartzo. Todos os corpos de prova, antes da oxidação, foram lixados com lixa de $\mathrm{SiC}$ com granulometria até 1200 e limpos em álcool em um banho ultrassônico. Medidas termogravimétricas foram realizadas usando uma termobalança da marca SARTORIUS com resolução de $10^{-5} \mathrm{~g}$. Os produtos de corrosão, ex. óxidos, foram analisados

* ${ }^{\circledR}$ Marca registrada da Special Metals Co.
Tabela I. Composições químicas (\% em massa) das ligas estudadas

\begin{tabular}{lccccccccc}
\hline \multicolumn{1}{c}{ Liga } & $\mathbf{F e}$ & $\mathbf{C r}$ & $\mathbf{N b}$ & $\mathbf{M o}$ & $\mathbf{T i}$ & $\mathbf{A l}$ & $\mathbf{S i}$ & $\mathbf{C}$ & $\mathbf{N i}$ \\
\hline $\begin{array}{l}\text { Inconel } \\
719\end{array}$ & 19,0 & 18,1 & 4,9 & 2,8 & 1,0 & 0,5 & 0,5 & 0,05 & bal. \\
$\begin{array}{l}\text { Inconel } \\
625\end{array}$ & 2,80 & 19,0 & --- & 8,9 & -- & 0,24 & $\mathbf{I}, 20$ & 0,01 & bal. \\
\hline
\end{tabular}

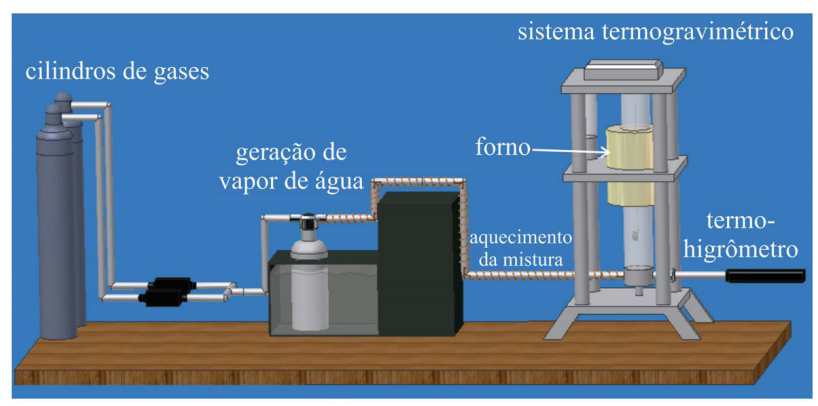

Figura I. Desenho esquemático do sistema de termogravimetria utilizado para oxidação em altas temperaturas com adição de vapor de água.

utilizando microscopia eletrônica de varredura (MEV), espectroscopia por dispersão de energia (EDS) e difração de elétrons retroespalhados (EBSD).

\section{RESULTADOS E DISCUSSÕES}

Medidas termogravimétricas mostram que a liga Inconel 625 apresenta resistência à oxidação superior à liga Inconel 718, como mostra a Figura 2 através do ganho de massa específico. Esta superioridade da liga Inconel $625 \mathrm{em}$ relação à liga Inconel 718 é provavelmente devida, entre outras diferenças, ao maior teor de Si na liga Inconel 625 que permite a formação de uma fina camada de $\mathrm{SiO}_{2}$ abaixo da camada externa de $\mathrm{Cr}_{2} \mathrm{O}_{3}$, veja Figura 2. Outros elementos como $\mathrm{Fe}$ e $\mathrm{Nb}$ que são diferentes nas duas ligas, podem ter influência no processo de oxidação, mas o Si é mais significativo em termos de formação de camada protetora.

Com o desenvolvimento da técnica de EBSD (hardware e software), análise microestrutural tornou-se mais eficiente em muitos aspectos, especialmente quanto à caracterização de contornos de grãos. A Figura 3 mostra uma comparação da microestrutura do Inconel 625 usando MEV. Na Figura 3a, usou-se detector de elétrons retroespalhados (BSE) onde se observa uma microestrutura com contraste de apenas duas cores: cinza clara e cinza escura. Por outro lado, a Figura $3 \mathrm{~b}$ mostra a mesma liga analisada utilizando EBSD, onde se observa detalhada microestrutura através de diferentes cores que representam diferentes orientações dos grãos.

Com a ajuda da técnica EBSD pode-se classificar os diferentes contornos de grãos a partir de suas orientações. A Tabela 2 mostra a medida da quantidade de grãos de baixo ângulo (=grãos especiais) presentes nas duas ligas estudadas. Como pode ser observado, a quantidade de grãos de baixo ângulo representa aproximadamente um terço do total. Adicionalmente, observa-se que a quantidade de 
grãos classificados como $\Sigma 3$ representa quase a totalidade dos grãos especiais.

Análise de superfície após oxidação mostra a formação de uma camada de $\mathrm{Cr}_{2} \mathrm{O}_{3}$ com pronunciada elevação desta camada ao longo dos contornos de grãos da liga. A formação da camada de $\mathrm{Cr}_{2} \mathrm{O}_{3}$ depende da difusão de átomos de $\mathrm{Cr}$ da liga para a superfície. Devido à maior difusão do $\mathrm{Cr}$ ao longo dos contornos de grãos da liga em comparação à difusão no grão, forma-se uma camada de $\mathrm{Cr}_{2} \mathrm{O}_{3}$ mais espessa na superfície, em regiões de contornos de grãos da liga (veja Figura $4 a$ ). Pode ser observado, também, que ao longo de alguns contornos de grãos, a camada de $\mathrm{Cr}_{2} \mathrm{O}_{3}$ apresenta mesmas características que em regiões do interior do grão, isto é explicado pelas propriedades dos contornos de grão. Contornos de grão de baixo ângulo exibem difusividade do Cr comparável à do grão, o que explica características similares das superfícies óxidas nestas regiões (veja Figuras 4a e 4b). Outro aspecto importante é a conectividade dos contornos de altos e baixos ângulos que pode influenciar no fluxo de elementos (Figura 4b).

A formação de uma camada de $\mathrm{Cr}_{2} \mathrm{O}_{3}$ na superfície da liga é muito importante para proteger o metal base da liga, o níquel neste estudo. No entanto, existem estudos que mostram que oxigênio pode penetrar na camada de $\mathrm{Cr}_{2} \mathrm{O}_{3}$ através de difusão ou através de poros ou trincas e entrar em contato com a liga reagindo com elementos menos nobres dando origem à chamada corrosão interna $[1,2]$.

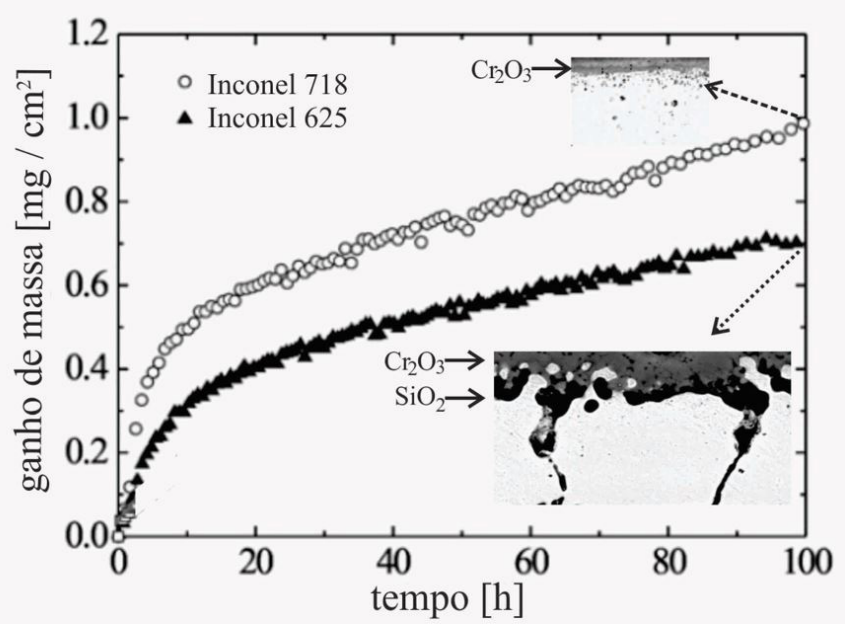

Figura 2. Medidas termogravimétricas das ligas Inconel 625 e Inconel 718 oxidadas a $850^{\circ} \mathrm{C}$ por $100 \mathrm{~h}$ na atmosfera descrita acima. Nota: marcador no eixo das ordenadas deve ser lido como vírgula.
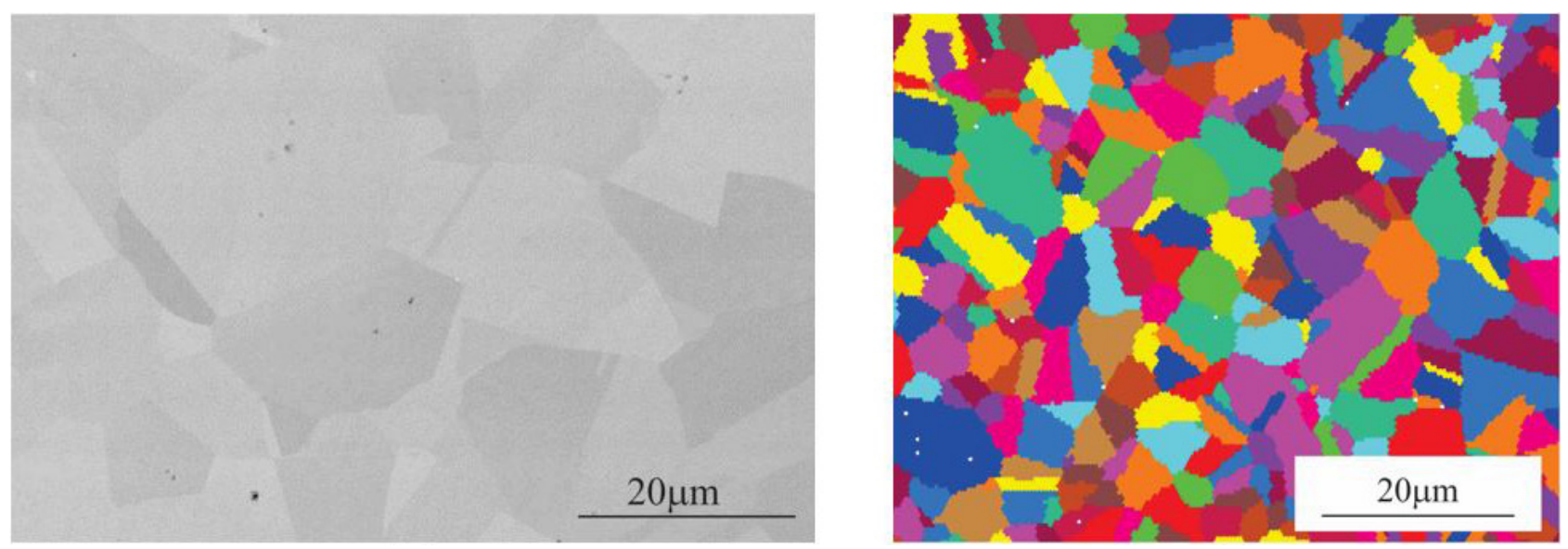

Figura 3. Observação microestrutural da liga Inconel 718 usando MEV. (a) através de detector elétrons espalhados (BSE) e (b) através da técnica de EBSD.

Tabela 2. Medida da quantidade (\%) de contornos de grão (CG) de baixo ângulo usando EBSD

\begin{tabular}{|c|c|c|c|c|c|c|c|c|c|c|c|c|c|c|}
\hline Tipo de CG & $\Sigma 3$ & $\Sigma 5$ & $\Sigma 7$ & $\sum 9$ & $\Sigma \mid \mathbf{I}$ & $\Sigma 13$ & $\Sigma 15$ & $\Sigma 17$ & $\Sigma 19$ & $\Sigma 21$ & $\Sigma 23$ & $\Sigma 25$ & $\Sigma 27$ & $\Sigma 29$ \\
\hline Inconel 7 I 8 & 34,1 & 0,5 & 0,6 & 1,5 & 0,4 & 0,2 & 0,3 & 0,3 & 0,3 & 0,3 & 0,2 & 0,4 & 0,3 & 0,1 \\
\hline Inconel 625 & 31,2 & 0,4 & 0,5 & 2,1 & 0,3 & 0,3 & 0,1 & 0,3 & 0,3 & 0,2 & 0,3 & 0,2 & 0,4 & 0,1 \\
\hline
\end{tabular}


Comumente, oxidação intergranular ocorre devido à rápida difusão do oxigênio nos contornos de grãos da liga, causando sua maior penetração nos contornos de grãos que no interior do grão. A Figura 5 mostra oxidação intergranular na liga Inconel 718 oxidada a $850^{\circ} \mathrm{C}$ por $100 \mathrm{~h}$. Pode ser observada a presença de uma camada externa que não é capaz evitar a oxidação interna. A zona de oxidação interna formada por $\mathrm{Al}_{2} \mathrm{O}_{3}$ pode ser diferenciada por uma fina zona homogênea de profundidade de em torno de $10 \mu \mathrm{m}$ e uma zona de oxidação intergranular com profundidade de em torno de $60 \mu \mathrm{m}$. É importante notar a presença de alguns contornos de grãos não oxidados como mostrado na Figura 5.

Análises de EBSD na seção transversal das ligas oxidadas mostram que contornos de grãos de baixo ângulo como $\circ \Sigma 3$, oferecem resistência à oxidação intergranular, enquanto que contornos de altos ângulos são atacados (veja Figura 6) devido à alta difusividade de oxigênio nestes contornos.

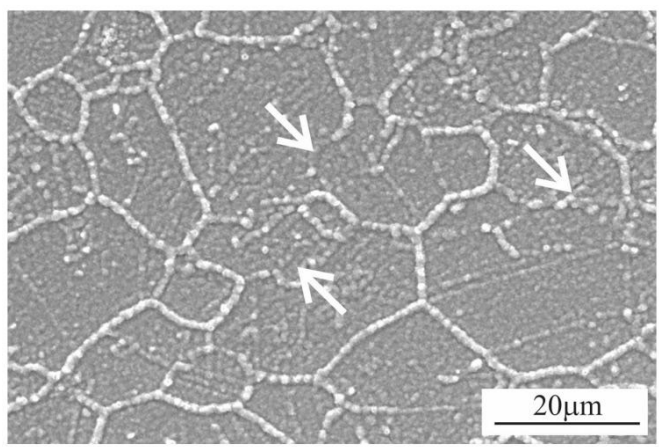

(a)

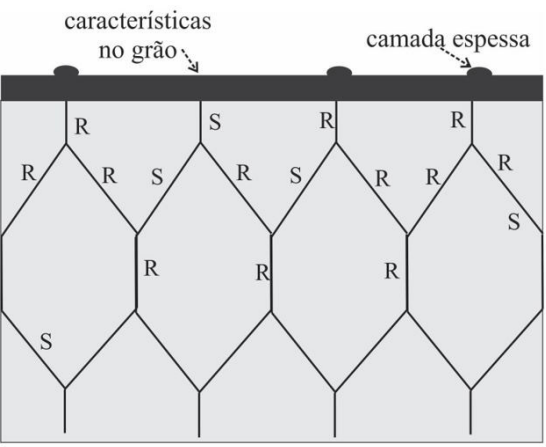

(b)

Figura 4. (a) Formação de $\mathrm{Cr}_{2} \mathrm{O}_{3}$ na superfície da liga Inconel 718 e (b) representação esquemática da conectividade dos diferentes tipos de contornos de grãos que podem influenciar no fluxo de $\mathrm{Cr}$ para a superfície da liga, bem como a difusão de oxigênio no interior da liga. $\mathrm{R}=$ random (contorno de alto ângulo) e $S$ = special (contorno de baixo ângulo).

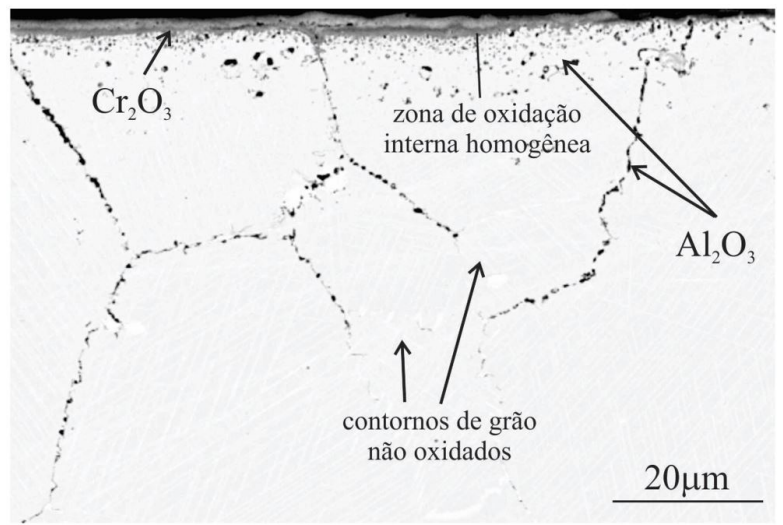

Figura 5. Oxidação intergranular na liga Inconel 718. Comportamento similar é observado na liga Inconel 625.

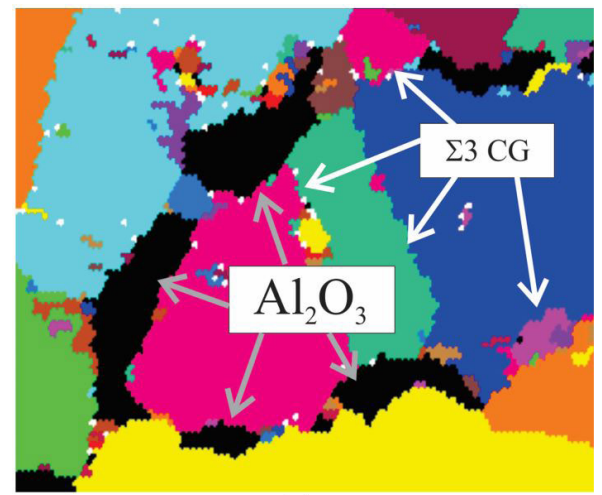

(a)

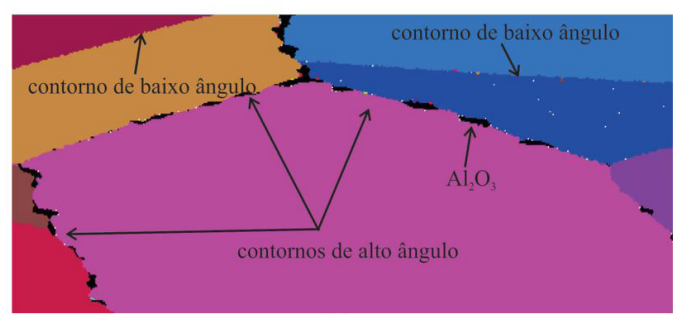

(b)

Figura 6. Análise de EBSD da oxidação intergranular na liga Inconel 625 a $850{ }^{\circ} \mathrm{C}$ durante $100 \mathrm{~h}$. (a) identificação do $\mathrm{Al}_{2} \mathrm{O}_{3}$ e (b) identificação dos contornos de grãos. 


\section{CONCLUSÕES}

O uso da técnica EBSD permite uma melhor descrição da microestrutura de ligas comparada com a análise clássica usando detector de elétrons secundários, pois uma análise estatística das características dos contornos de grãos é possível com o uso do EBSD. O presente estudo demonstra a importância das características dos contornos de grãos para a formação de uma camada externa protetora.
Similarmente, a formação de uma zona de oxidação intergranular é influenciada pela presença de grãos de baixo ângulo, bem como pela conectividade entre os ângulos de altos ângulos. Foi demonstrada a formação de $\mathrm{Al}_{2} \mathrm{O}_{3}$ ao longo de contornos de altos ângulos e nenhuma evidência de oxidação de contornos de baixos ângulos como $\Sigma 3$. Este estudo descreve as bases para trabalhos em direção à engenharia do contorno de grão objetivando a melhoria da resistência à corrosão em altas temperaturas.

\section{REFERÊNCIAS}

I Douglas DL. A critique of internal oxidation in alloys during the post-Wagner era. Oxidation of Metals. 1995;44:81III.

2 Trindade VB. Corrosão de ligas metálicas em altas temperaturas: high-temperature corrosion of metallic alloys. Vila Velha: Above Publicações; 2014.

3 Trindade VB, Krupp U, Wagenhuber PEG, Christ H-J. Oxidation mechanisms of $\mathrm{Cr}$-Containing steels and Ni-Base alloys at high temperatures - part I: the different role of alloys grain boundaries. Materials and Corrosion. 2005;56:785-790.

4 Randle V. Refined approaches to the use of the coincidence site lattice. JOM. 1998;2:56-59.

5 Watanabe T. Approach to grain boundary design for strong and ductile polycrystals. Res Mechanica. 1984; I : $47-84$.

6 Lehockey EM, Palumbo G, Lin P, Brennenstuhl AM. On the relationship between grain boundary character distribution and intergranular corrosion. Scripta Materialia. 1997;36:12 II-12 I8.

7 Was GS, Thaveeprungsripon DC, Crawford DC. Grain boundary misorientation effects on creep and cracking in ni-based alloys. JOM. 1998;2:44-49.

8 Trindade VB, Krupp U, Wagenhuber PE-G, Virkar YM, Christ H-J. Studying the role of the alloy-grain-boundary character during oxidation of ni-base alloys by means of the EBSD technique. Materials at High Temperatures. 2005;22:3I-36.

9 Lehockey EM, Palumbo G. On the creep behaviour of grain boundary engineered nickel. Materials Science and Engineering A. 1997;237:168-172.

I0 Lin P, Palumbo G, Erb U, Aust KT. Influence of grain boundary character distribution on sensitisation and intergranular corrosion of alloy 600. Scripta Metallurgica et Materialia. 1995;33: I387-I 392.

Recebido em: 30 Maio 2016

Aceito em: 20 Fev. 2017 